Proc. 15th Int. Conference on Defects Recognition, Imaging and Physics in Semiconductors, Warsaw, Poland 2013

\title{
Parallel Optical Profilometry Assisted Conception of an Opto-Electronic Illuminator Using a VCSEL and Diffractive Optics
}

\author{
P.C. Montgomery*, B. Serio and P. Twardowski \\ Laboratoire des Sciences de l'Ingénieur, de l'Informatique et de l'Imagerie (ICube) \\ UDS-CNRS, UMR 7357, 23 rue du Loess, 67037 Strasbourg, France
}

\begin{abstract}
Micro-opto electromechanical systems are of growing importance in the development of new miniaturised projection, imaging and detection technologies. Because of the increasing complexity of construction involving layers of microelectronics, optoelectronics and micro-optics, different types of defects can appear that need to be understood and controlled in order for the miniaturised system to operate correctly within the given specifications. We have recently developed a miniaturised structured light (micro-opto electromechanical systems) projector consisting of a multilayer sandwich made of a first layer of 3 bars of 12 VCSEL laser sources, a second layer of Fresnel collimating lenses (diffractive optical elements) and a third layer of Fourier diffractive optical elements to produce the structured light pattern. The main defects that had to be controlled were alignment errors, the first ones being between the emitting surface and the collimating lenses and the second one concerning the lateral alignment of the photomasks used to produce the 4 levels of the Fourier elements. We demonstrate how 3D geometrical shape characterization of the emitting structures and diffractive optical elements surface structures using coherence scanning interferometry played a major role in the conception and fabrication of a prototype micro-optoelectromechanical projection system to understand the source of the alignment errors and to minimise them.
\end{abstract}

DOI: $10.12693 /$ APhysPolA.125.957

PACS: 85.85.+j, 42.55.Px, 42.40.Eq, 42.87.Bg, 68.35.bg

\section{Introduction}

The integration of micro-optics, micromechanics and microelectronics on the same chip has led to the new technology known as micro-opto electromechanical systems (MOEMS). The potential for embossed replication and batch processing makes them highly attractive for use in new miniaturised projection, imaging and detection technologies. For example, digital micro-mirror arrays (DMD) are widely used in present day video projectors. One of the applications of such projectors for metrology is in the field of $3 \mathrm{D}$ shape measurement of macroscopic objects using fringe projection [1]. The requirements of medical or industrial inspection are for higher speed and smaller systems. One way of miniaturising the fringe projection system is to use fixed fringe patterns that are switched using the illumination, for example with separate LED's combined with arrays of microlenses and microslides [2].

Going a step further in size reduction is possible using a completely integrated system of light sources and micro-optics in a single MOEMS system. We recently made this step in developing a miniaturised structured light MOEMS projector for 3D shape measurement having a projection head of only a few $\mathrm{mm}$ in size. Successive pattern projection is made by using switched VCSEL

*corresponding author; e-mail: paul.montgomery@unistra.fr laser sources combined with diffractive optical elements (DOE) [3]. The MOEMS projector consists of three arrays of 12 vertical-cavity surface-emitting lasers (VCSEL) together with two planar static DOE arrays made in quartz. The first DOE consists of Fresnel-type elements for collimating each laser beam and the second DOE serves to produce different structured light patterns. The availability of 36 lasers means that 36 different patterns could in principle be incorporated into the same system. In our particular case, we used just 8 of the channels to produce the phase shifted gratings and modified Gray codes for 3D measurement [3].

Because of the increasing complexity of construction involving layers of microelectronics, optoelectronics and micro-optics, different types of defects can appear that need to be understood and controlled in order for the miniaturised system to operate correctly within the given specifications. We have previously demonstrated the use of coherence scanning interferometry (CSI) in the characterization of the topography of a miniaturised MOEMS FT spectrometer [4] and of DOEs made using photoablation of an intermediate resist layer [5]. The particularity of CSI is that it allows deep characterisation $(<150 \mu \mathrm{m})$ with $\mathrm{nm}$ axial sensitivity on smooth surfaces over wide fields $(\mathrm{mm})$ [6]. The main defects appearing in the presently described MOEMS projector that needed to be understood and controlled were alignment errors, axially between the surfaces of the laser emitting sources and the Fresnel collimating lenses and laterally concerning the different photomasks used to produce the DOE structures. In this paper we demonstrate how parallel op- 
tical profiling using CSI has played a vital role to ensure that the microsystem design and fabrication has sufficient precision.

The first challenge was to align the laser sources to within $1 \mu \mathrm{m}$ axially with the focal points of the linear arrays of collimating DOE Fresnel lenses. The bought-in VCSEL laser components showed variations in the heights of the laser emission spots with respect to the substrate between the different models from different suppliers that were tested. This made it necessary to measure the actual heights of the emission areas, firstly to check that the values were uniform for a given component and secondly so as to be able to adapt the heights of the mounting pillars of the DOEs.

The second challenge concerned the quality of the fabrication of the Fourier elements which were designed to have 4 step levels to achieve the 4 levels of phase so as to improve the efficiency of the elements [7]. Such a design increases the efficiency by a factor of 2 compared with a two level mask. To produce the 4 levels, two stages of photomasks in the surface etching procedure are used. This required two separate optical photomasks that needed to be aligned laterally to submicron precision. Although the photomask equipment used had only a lateral precision of $1 \mu \mathrm{m}$, by using carefully designed alignment features on the masks and by fabricating sev- eral components, suitably accurate DOEs displaying little residue on edge features could be found by CSI.

\section{Design of the MOEMS projector}

The MOEMS projector produces a series of successive fringe patterns that combine the unambiguous and robust Gray code method with the high resolution of the phase shifting technique for more accurate 3D measurement of macroscopic objects [3]. The design of the MOEMS is based on a three layer system consisting of VCSEL together with two planar static DOEs arrays made in quartz, the first to collimate the laser beams and the second to produce the structured light pattern (Fig. 1a) [3].

Two laser sources were tested. The first was the TSA-8B12-000 VCSEL 3 bar array of 12 lasers each from TrueLight, emitting at a wavelength of $850 \mathrm{~nm}$ (Fig. 2). The characteristics are given in Table I. The interest of such a laser source is the availability of a large number of laser sources in a compact array design and the low cost $(<50 € /$ bar $)$. The second VCSEL laser source was the 850-2093-002 VCSEL single array of 12 lasers from Finisar (Fig. 2b) (Table I).

The characteristics of the VCSEL lasers.

TABLE I

\begin{tabular}{c|c|c|c|c|c|c|c}
\hline \hline $\begin{array}{c}\text { Laser } \\
\text { model }\end{array}$ & $\begin{array}{c}\text { Number } \\
\text { of lasers }\end{array}$ & $\begin{array}{c}\text { Emission } \\
\text { wavelength }\end{array}$ & $\begin{array}{c}\text { Output } \\
\text { power }\end{array}$ & $\begin{array}{c}\text { Chip size } \\
\text { of one array }\end{array}$ & $\begin{array}{c}\text { Size of one } \\
\text { VCSEL }\end{array}$ & $\begin{array}{c}\text { Active } \\
\text { area }\end{array}$ & $\begin{array}{c}\text { Measured } \\
\text { beam } \\
\text { divergence } \\
\text { (FWHM) }\end{array}$ \\
\hline $\begin{array}{c}\text { TrueLight } \\
\text { TSA-8B12-000 } \\
\text { Finisar } \\
850-2093-002\end{array}$ & $3 \times 12$ & $850 \mathrm{~nm}$ & $1.5 \mathrm{~mW}$ & $2970 \mu \mathrm{m} \times 320 \mu \mathrm{m}$ & $250 \mu \mathrm{m} \times 300 \mu \mathrm{m}$ & $\begin{array}{c}20 \mu \mathrm{m} \\
\text { diameter } \\
20 \mu \mathrm{m} \\
\text { diameter }\end{array}$ & $16^{\circ}$ \\
$11^{\circ}$
\end{tabular}

The first DOE consists of Fresnel-type elements for collimating each laser beam [7]. These are made of 8 diffractive elements (Fresnel lenses: L1, L2...L8), each containing $3 \times 12$ lenses with a focal length of $1 \mathrm{~mm}$, to be placed just above the VCSEL lasers. The second DOE consists of Fourier DOE computer generated holograms (CGH) for producing the different structured light patterns. The availability of 36 lasers means that in principle, 36 different patterns could be incorporated into the same system. In our particular system, we just used 8 lasers in one array to produce the phase stepped sinusoidal fringes and the modified Gray codes [3].

The DOEs were designed in-house using the finite element method (FEM) and the radiation spectrum method (RSM). They were made using photolithography in a two-step process with two photomasks and etching with reactive ion etching (RIE) of fused silica quartz (thickness $=525 \mu \mathrm{m}$ ). Two step levels were used at the edges of the elements and 4 steps at the centre. The first step height is calculated from $h_{1}=\lambda / 2(n-1)$ and the second from $h_{2}=\lambda / 4(n-1)$. For a wavelength of $850 \mathrm{~nm}$ and with $n=1.5$ for quartz, this gives $h_{1}=850 \pm 42 \mathrm{~nm}$ $( \pm 5 \%)$ and $h_{2}=425 \pm 21 \mathrm{~nm}( \pm 5 \%)$ (Fig. 3a).

\section{Zygo measurement system and data analysis}

The microscope system used to measure the MOEMS was a Zygo NewView 7200 interference microscope using scanning white light interferometry [6] and equipped with $10 \times$ and $50 \times$ Mirau interference objectives, a $150 \mu \mathrm{m}$ piezoelectric vertical stage and a digital $\mathrm{B} \& \mathrm{~W}$ camera. Zygo propriety image processing and analysis software was used to study the 3D measurement data by means of roughness parameters, line profiles and $3 \mathrm{D}$ views. The 


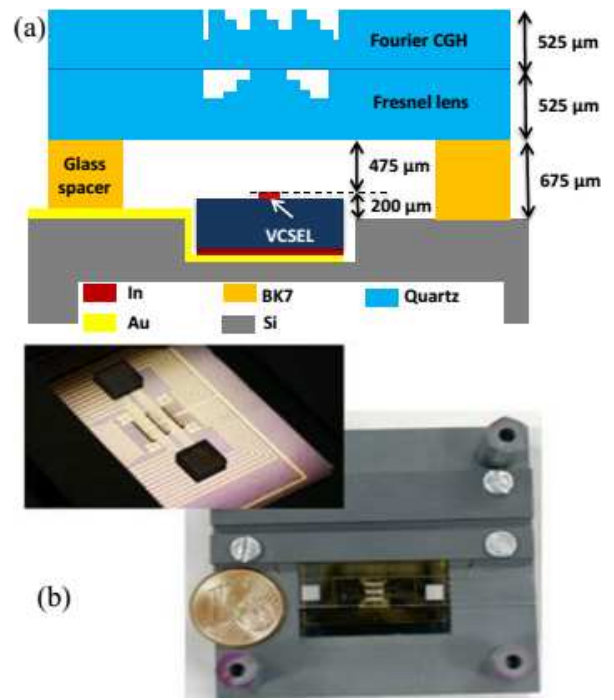

Fig. 1. Miniature MOEMS fringe projector: (a) schematic layout of MOEMS design, (b) finished MOEMS with 1 cent $(\Theta)$ coin and close-up of VCSEL array (inset).
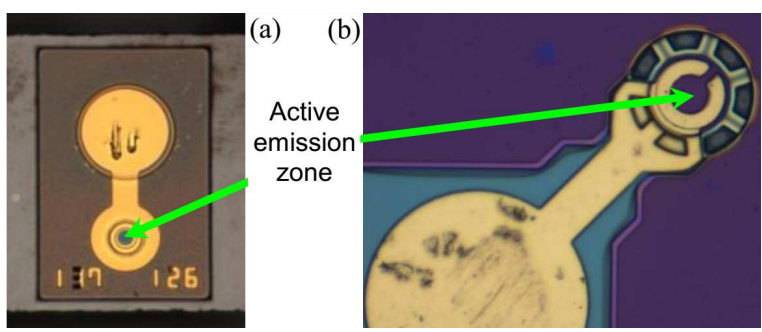

Fig. 2. Details of the VCSEL laser sources tested: (a) one laser from the TrueLight VCSEL model, (b) one laser from the Finisar VCSEL model.

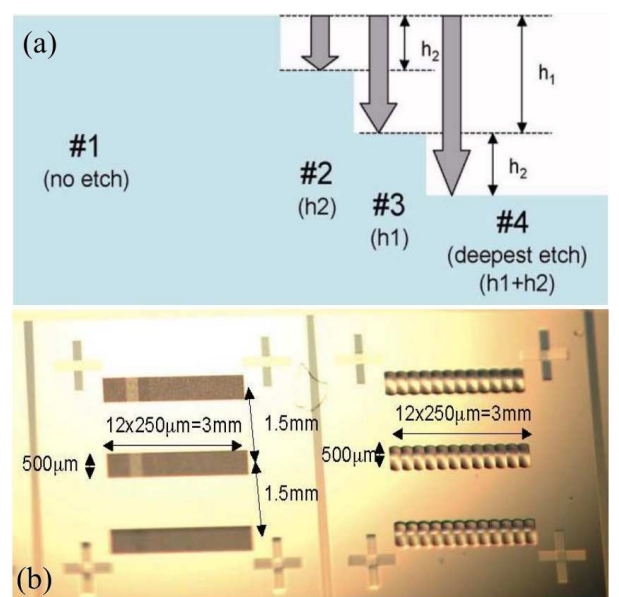

Fig. 3. Details of the DOEs: (a) details of two etching depths, $h_{1}$ and $h_{2}$, to produce the 4 step heights in the DOEs, (b) uncut Fourier (left) and Fresnel (right) DOEs. characteristics of the imaging system and objectives are shown in Table II.

TABLE II

The characteristics of the Zygo NewView 7200 microscope.

\begin{tabular}{c|c|c|c|c|c}
\hline \hline $\begin{array}{c}\text { Objective } \\
\text { magnifi- } \\
\text {-cation }\end{array}$ & $\begin{array}{c}\text { Numerical } \\
\text { aperture }\end{array}$ & $\begin{array}{c}\text { Lateral } \\
\text { optical } \\
\text { resolution } \\
{[\mu \mathrm{m}]}\end{array}$ & $\begin{array}{c}\text { Max } \\
\text { image } \\
\text { size } \\
{[\text { pixels }]}\end{array}$ & $\begin{array}{c}\text { Sample } \\
\text { pixel } \\
\text { size } \\
{[\mu \mathrm{m}]}\end{array}$ & $\begin{array}{c}\text { Max field } \\
\text { of view } \\
{[\mu \mathrm{m}]}\end{array}$ \\
\hline $10 \times$ & 0.3 & 0.95 & $640 \times 480$ & 1.1 & $700 \times 530$ \\
$50 \times$ & 0.55 & 0.52 & $640 \times 480$ & 0.22 & $140 \times 110$
\end{tabular}

The measurement data was also analysed using a program developed in-house using Mathematica to study the lateral eccentricity of the separately etched levels of the Fresnel lenses as well as a statistical analysis of the four etched heights and the resulting material residue lying outside the 4 levels required.

\section{Results of measurements of vertical alignment of VCSEL surfaces with Fresnel lenses}

The first challenge in the design and assembly of the MOEMS was to align the laser sources axially to within $1 \mu \mathrm{m}$ with the focal points of the linear array of collimating DOE Fresnel lenses. This was done with glass spacers cut to the correct thickness (Fig. 1a) and glued in place using epoxy resin. With the different models of bought-in VCSELs having laser emission spots varying in height with respect to the substrate, it was necessary to measure the actual heights of the emission areas so as to be able to adjust the approximate spacer height for a given laser array.

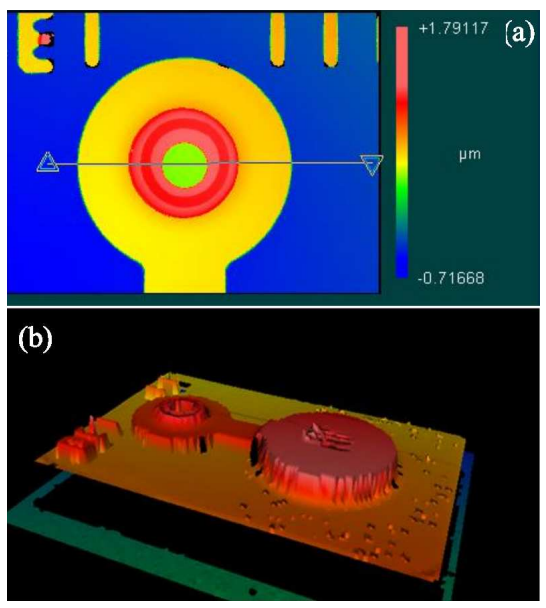

Fig. 4. Results of optical profilometry of a single TrueLight VCSEL laser: (a) colour coded height image of an individual laser emission zone (shown in green, $20 \mu$ m diameter), (b) 3D view of one VCSEL (rectangle $320 \mu \mathrm{m} \times 220 \mu \mathrm{m})$.

Results of topographic measurements of both models of VCSEL lasers are shown in Fig. 4 (TrueLight model) 


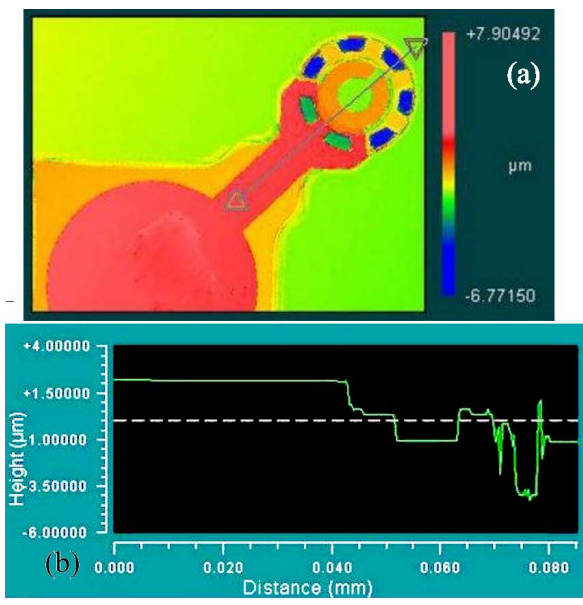

Fig. 5. Results of optical profilometry of a single Finisar VCSEL laser: (a) colour coded height image of laser source, (b) 2D profile of laser emission zone.

and Fig. 5 (Finisar model). Final alignment of the focal points of the Fresnel lenses was performed by adjusting the height using epoxy resin while analysing the diameter of the projected collimated beams.

\section{Results of measurements of horizontal alignment of photomasks for 4 level etching of DOEs}

The second challenge concerned the quality of the fabrication of the DOEs due to the use of two stages of photomasks in the surface etching procedure to make the 4 step levels necessary to achieve the 4 levels of phase to improve their efficiency. This required two separate optical photomasks that needed to be aligned laterally to submicron precision. Although the photomask equipment used had a lateral alignment uncertainty of only $1 \mu \mathrm{m}$, by using carefully designed alignment features on the masks and by fabricating several components, the $3 \mathrm{D}$ optical profiling served to find the components having a suitably accurate DOE displaying little residue on edge features.

The results in Fig. 6 show the characterization by optical profilometry of a Fresnel DOE using a poorly aligned photomask. The topographic results in Fig. 6a show up the insufficient quality of the $\mathrm{DOE}$. The $2 \mathrm{D}$ profile in Fig. $6 \mathrm{~b}$ reveals in detail the presence of the four required etched levels together with residue material along several edges. Normalising the heights in Fig. 6c, the eccentricity of the two etched structures becomes visible.

Performing a statistical analysis of the 3D data, a histogram of the distribution of heights in Fig. $6 \mathrm{~d}$ reveals the four required etched levels as the four highest peaks, together with a non-negligible series of smaller peaks in between corresponding to excess residue material. In comparison, the results in Fig. 7 show the characterization by optical profilometry of a Fresnel DOE using a well aligned photomask. The topographic results in Fig. 7a
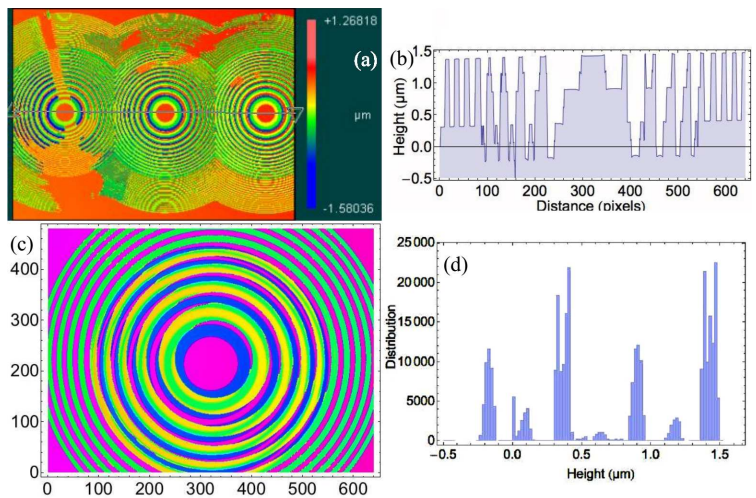

Fig. 6. Results of optical profilometry of a Fresnel lens made using a poorly aligned photomask: (a) colour coded height image of 3 Fresnel lenses, (b) 2D horizontal profile in centre of (a), (c) normalised heights of Fresnel lens to show eccentricity of 4 etched levels, (d) histogram of height distributions for whole image showing the 4 required etch levels (highest peaks) and excess residue in between (lowest peaks) due to photomask misalignment.

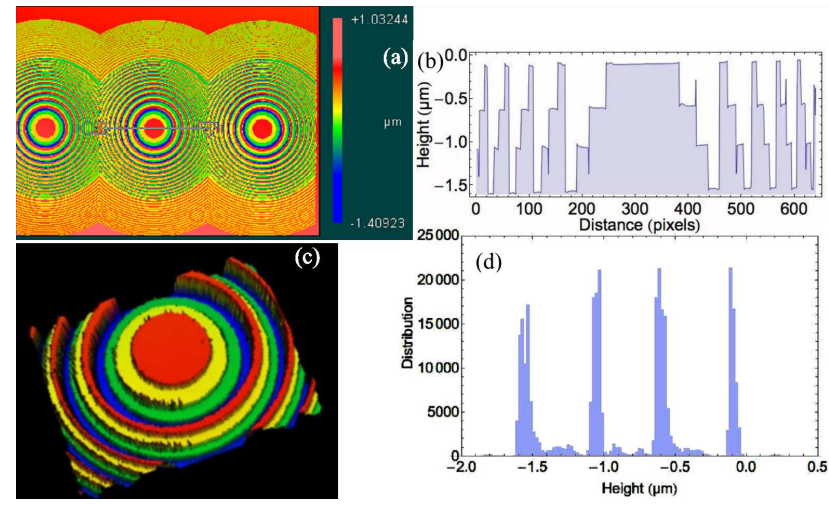

Fig. 7. Results of optical profilometry of a Fresnel lens made using a well aligned photomask: (a) colour coded height image of 3 Fresnel lenses (10× objective), (b) 2D horizontal profile in centre of (a), (c) 3D view of a single Fresnel lens (50 $\times$ objective), (d) histogram of height distributions for whole image showing the 4 required etch levels (highest peaks) and slight residue in between (lowest peaks) due to good photomask alignment.

and c clearly show up a good quality DOE. The $2 \mathrm{D}$ profile in Fig. $7 \mathrm{~b}$ reveals in detail the presence of the four required etched levels together with a small amount of residue material.

The presence of the four clearly defined highest peaks in the histogram of the distribution of heights in Fig. $7 \mathrm{~d}$ confirms the overall quality of the four required etched levels. The fact that the peaks in between are very small shows that the residue material is negligible.

Because of the circular symmetry of the Fresnel lens DOEs, the eccentricity of the two alignment patterns could be used as an indication of the quality of the resulting DOE. In the case of the Fourier CGH, the lack 


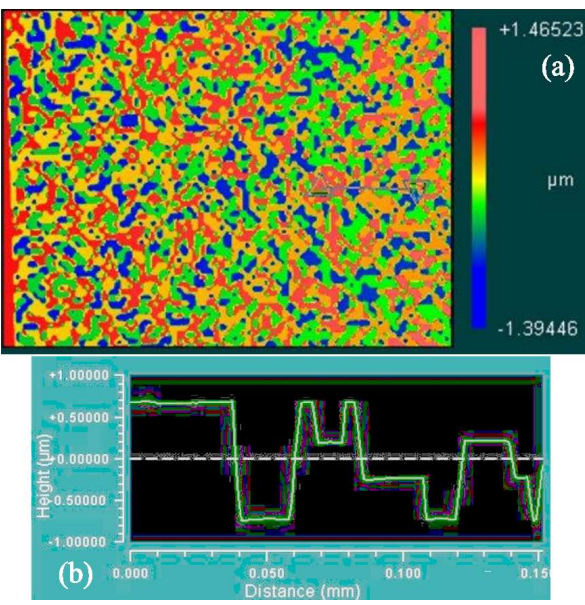

Fig. 8. Results of optical profilometry of a Fourier CGH DOE made using a well aligned photomask: (a) colour coded height image $(50 \times$ objective), (b) 2D profile from Fig. 1 showing 4 step levels.

of lateral symmetry in the topographic image (Fig. 8a) meant that the only ways of studying the quality was to look at individual 2D profiles (Fig. 8b) and the histogram of the distribution of the four etched levels in order to be able to select $\mathrm{CGH}$ DOEs with suitable quality.

\section{Discussion and conclusions}

The first prototype of a miniaturized MOEMS structured light projector has been developed using bought in VCSEL laser arrays and a two stage optical system for collimating the laser beams and producing the structured light pattern. The projection head is only a few $\mathrm{mm}$ in size. An essential part of the success of its design and fabrication was the use of 3D metrology using an optical profiler based on interference microscopy (the Zygo NewView 7200 model). This measurement system was used at two critical stages of the metrology. The first was to measure the heights of the laser emission zones of the VCSEL lasers so as to be able to make appropriately high spacers in between the laser array and the Fresnel collimating DOE. The second stage was in the measurement of different DOEs etched in quartz to find a spec- imen with a suitable quality of the four etched heights showing that alignment of the two photomasks was correct. The study of roughness, $2 \mathrm{D}$ profiles, $3 \mathrm{D}$ views, normalized height images and histograms of the distribution of heights allowed a suitable DOE to be found for the MOEMS system. The optical profiler enabled several different types of metrological information and details of the defects of the different parts to be understood and minimized to make an improved system.

The next difficulties to overcome will be to miniaturize the connections to the VCSEL lasers as well as that of the packaging. Future designs will also involve the use of laterally sub wavelength optical elements, which although requiring only a single mask that will eliminate any alignment errors, will require subwavelength structuration and metrology [2]. In combination with plastic DOEs giving phase variations from a structured surface relief, compact, programmable and low cost MOEM projectors will then be able to be made.

\section{Acknowledgments}

The authors would like to acknowledge the financial support of the "TriDif" Oséo project.

\section{References}

[1] S.S. Gorthi, P. Rastogi, Opt. Las. Eng. 48, 133 (2010).

[2] S. Heist, M. Sieler, A. Breitbarth, P. Kühmstedt, G. Notni, Proc. SPIE 8788, 878815 (2010).

[3] P. Twardowski, B. Serio, V. Raulot, M. Guilhem, Proc. SPIE 7716, 77162I (2010).

[4] P.C. Montgomery, D. Montaner, O. Manzardo, M. Flury, H.P. Herzig, Thin Solid Films 450, 79 (2004).

[5] M. Flury, A. Benatmane, P. Gérard, P.C. Montgomery, J. Fontaine, T. Engel, J.P. Schunck, Opt. Eng. 41, 2407 (2002).

[6] J. Schmit, J. Reed, E. Novak, J.K. Gimzewski, Opt. A, Pure Appl. Opt. 10, 064001, (2008).

[7] V. Raulot, P. Gérard, B. Serio, M. Flury, B. Kress, P. Meyrueis, Opt. Expr. 18, 17974 (2010). 